\section{Click location and syntactic structure}

\section{M. HOLMES, * University of Melbourne, Parkville, Victoria 3052, Australia and}

K. I. FORSTER, Monash University, Clayton, Victoria 3168, Australia

Four experiments are reported in which Ss had to judge the location of clicks superimposed on recorded sentences. The first experiment showed that the accuracy of locating the clicks was a function of the position of the click in the constituent structure, the greatest accuracy being for clicks at major clause boundaries. The second experiment showed that this effect was independent of migration, i.e., the tendency for judgments to be displaced towards the major clause break. In the third experiment, it was shown that the requirement that $S$ reproduce the sentence did not influence the response distribution. Finally, in the fourth experiment, a small but significant trend for location accuracy to decrease with decreasing separation of the click from the major break was found. However, this trend was much smaller than the differences in accuracy for various positions in the constituent structure. It was concluded that click location accuracy can be used as an index of perceptual processing load during recognition of individual sentences.

In experiments by Fodor and Bever (1965), Garrett, Bever, and Fodor (1966), Bever, Kirk, and Lackner (1969), Bever, Lackner, and Kirk (1969), and Bever, Lackner, and Stolz (1969), Ss were required to judge the location of an extraneous signal (usually a click) which occurred during a recorded sentence. The results showed that Ss systematically mislocated the clicks towards the nearest major clause boundary within the sentence. This "migration" or "attraction" of the judgments of click location has been interpreted as demonstrating that the clause is a major perceptual unit in sentence recognition. According to Bever, Lackner, and Kirk (1969) and Bever (1970), the click migration effect shows that the listener initially segments the input into those sequences which correspond to a sentence in the underlying structure of the sentence. As well as obtaining the migration effect, Fodor and Bever (1965) found that clicks at major constituent boundaries were more accurately located than were the clicks adjacent to such boundaries. They took the results to be merely a consequence of their main conclusion about click migration, i.e., Ss were less likely to mislocate clicks at major breaks because the clicks would not be attracted away from such positions.

It can be argued that variations in the accuracy of click location are not just a byproduct of the click migration effect but are a result of differential interference from processing the sentence structure in the immediate context of the click. Using a click

*Now at the Department of Psychology, Building E10 Massachusetts Institute of Technology, Cambridge, Massachusetts 02139 . detection task, Holmes and Forster (1970) showed that reaction time to clicks varies at different locations in the sentence structure: the fastest reactions to clicks occurred at major clause breaks. This effect is presumably a result of differences in attention required by concurrent sentence processing, suggesting that there is a temporary lull in processing activity at the major syntactic break. Such variations in processing load may also underlie Fodor and Bever's (1965) accuracy effect of click location. At points where $\mathrm{S}$ is able to react relatively rapidly to a click, he may also be able to remember its location more accurately.

While these remarks about click location accuracy are not incompatible with the interpretation of the click migration effect proposed by Fodor and Bever (1965), they provide a different perspective for viewing the processes operating in the click location task. Furthermore, click location accuracy seems much more straightforward as a measure of performance than does click migration. Criteria for determining whether click migration has occurred are somewhat arbitrary, a fact which is evidenced by the variety of procedures used to assess the magnitude of the effect. The measure of click location accuracy is simply whether the judgment is correct.

The following experiments aimed to explore the use of click location accuracy as an index of processing load during sentence recognition. Specifically, it was intended to see whether Fodor and Bever's (1965) result, that accuracy of click location is greater at major constituent breaks than within major constituents, is a general one or is specific to conditions where migration occurs. That is, the accuracy of locating clicks occurring near the major break may have been reduced simply by the migration effect and not by increased sentence processing load. In that case, the lower error rate for locating clicks at the major break might only reflect lowered accuracy at the adjacent positions. To eliminate this possibility, it is necessary to demonstrate that differences in accuracy can occur independently of migration effects. It seems reasonable to expect that migration effects would be less likely to occur for clicks placed far from the break instead of the one-to-four-syllable distances used in all the studies of click migration. Hence, in comparing the accuracy of locating clicks at the major break with that of clicks at other positions in the structure of the sentence, we used click positions which were at a considerable distance from the break. If accuracy of click location indicates the processing load imposed by those parts of the sentence surrounding the click, then accuracy should still be dependent on the position of the click in the syntactic structure of the sentence, even in the absence of a click migration effect.

GENERAL DESIGN AND PROCEDURE OF EXPERIMENTS

In each experiment, a randomly ordered list of sentences was recorded on one track of a stereophonic tape recorder. A short burst of noise, a "click," was recorded on the other tape track. In Experiments 1 and 2 the duration of the click was about 80 msec, while in Experiments 3 and 4 the duration of the click was 1 msec. Owing to the smoothing effect of the headphones, the 1-msec click was effectively about 20 msec, and, in fact, there appeared to be little difference subjectively between the two click lengths. The intensity of the clicks was adjusted so that it was approximately equal perceptually to the loudest speech sound. None of the clicks was located within a word; instead, each click was placed just at the end of a word so that it did not occur during the acoustic pause possible between words. The energy level of the two tape tracks was analyzed, using a two-channel pen recorder, and the results verified the assumption that the clicks did not occur during acoustic pauses.

The experiments were designed so that repeated measurements were obtained from each $\mathbf{S}$. Each $\mathbf{S}$ heard every one of the sentences, which were presented in one of four orders arranged from a Latin square design. The sentences in each experiment varied in syntactic structure, except for one important constraint: each 


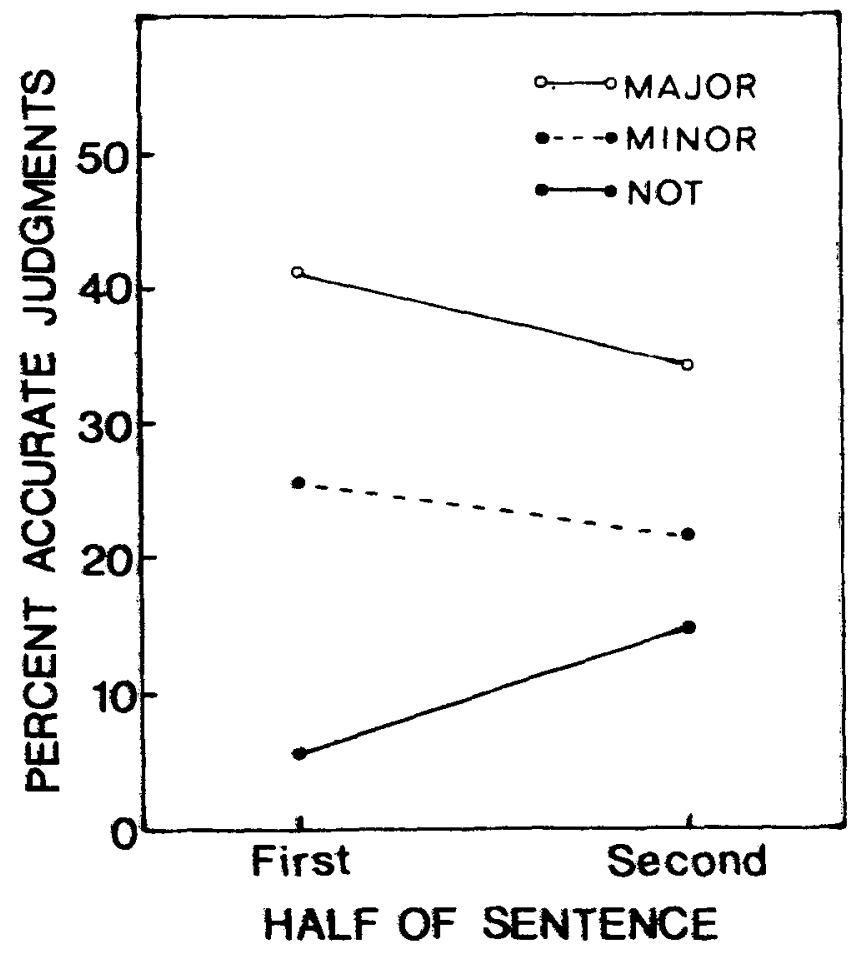

Fig. 1. Percentage of accurate click location judgments in Experiment 1 as a function of position of the click serially in the sentence and of position of the click in the constituent structure (at a major clause break, at a minor break, and not at a break).

sentence had a major surface structure boundary where no constituents except the matrix $S$ itself were interrupted. This boundary divided the sentence into two clauses. ${ }^{2}$ The sentences were either coordinate clause constructions or adverbial clause constructions with the adverbial clawse either first or econd. In order to avoid stereoty ped responding owing to monotcay of the sentences, the semantic content wres rande as raried and interesting porible some examples are: Until the Foneism Office states the facts, we will not believe the wild rumors; The young doctor cunnot join the team if the teat's newilks are not atiofactory: The crowd cheered, as the cast of the phy neturmed on stage for an encone; Tongues of flume licked up from the buming butdine. and spariss shot into tive sty.?

The stimali mere presented through headphones, and presentation of centences in one enr and clicls in the other was altemated ackoss the onders. Ss were instructed to wite the sentence out as accurately as poosible and to plnce a slash sxark to indicate as accurately as pocible the location of the click. So were given several practice examples. All the So used in each experiment were native speakers of English.

Clicks were placed at three positions in the constituent structure of sentenoes. Fimst, clicks were located at the major clanse break which separated coordinate clawses or the adverbial clawse from the main clawe. In moat andyes of surface constituent structure, no constituents except the matrix $\mathbf{S}$ itoelf axe interrupted at this point. Secoudly, clicks were placed at divicions within one of the mojor clauses, either at the boundiries of embediled clanses or between phuswes. These locutions were comidered to be at "minor" breaks in the constituent structure. Thirdly, clich were plood at points either within simple noun phrases or within the anxiliery expourion of vert plures. Since these ciek bentions interrupt a bye number of condituents, they were clawified as being not at a break in the structure. No attempt was made to specify the namber of constituents interupted by the clicks in the bither two condition becuse a particular surface structure analyos scocriting to different theoretical orientation. The three kinds of click positions are shown in the following example: When they arrived/, they found/ the woman was screaming and in a state of hy steria. In our clasification, the finst bish represents a click at a mior clawse break, the second click is at a minor break, and the third click is not at a break. senteroe my be given different
Two hypotheses were tested: (1) Click location judgments should be more accurate at a major clause break than either at a minor break or not at a break. This predietion is based on Holmes and Forster's (1970) conclussion that attention to sentence processing is reduced at major clatse breaks compared with other parts of the sentence; (2) location accuracy should be greater for clicks at a minor brenk than for clichs not at a break if a decrease in sentence processing load also oceurs at minor breales in the constituent structure. In addition, it was predicted that migration mould not occur because the relevant click positions were so far awry from the major clause break.

\section{Design}

The 24 stimulus sentences were the same as thowe listed in Holmes and Fonster (1970). The position of the clict in the constituent structure was: the main treatment condition (constituent break type): the click was either at a mojor clause break, at a minor breal, or not at a break. For the butter two conditions, the average seprrations of the click from the major clanse boundary were 6.3 and 16.9 syllables, respectively. Clicks were abo located at different serial poxitions in the entence: either in the fint half of the sentence (earty) or is the second half of the ventence (late). As a form of internal replication, two sets of stimuli were prepared. The enme 24 sentences were recorded twice, with cticks placed in different positions, and ench set of sentences was presented to a separate group of 20 $\mathrm{se}^{3}$

\section{Subjects}

The Sh mere 40 volunbees from a underyreduate preholosy couse at the Univeasity of Meltooume.

\section{Recults}

The sum of each Sts socurite click bentions for the four sentences in each oondition wio obtrined The location scournes wevis over all $S$ s axe shome as pencentreos in Fic 1. A four-wy amalyis of variance ras performed on the data (S. by Groups: by Coatituent Break Type by Serial Porition). The eum of pruares (SS) for the condituent break type factor was divided into two components correoponding to the two orthogonal predicted contrasts (Flays, 1963). Setting the Type I error rate at $\alpha=.05$, both contrasts were significant. More accurnte judgments were made for clicles at a imjor breal than for click either at a minor break or not at a beenk, with $P(1,76)=$ 35.75; more accurate judganents were mede for clicks at a mincr break then for clicks not at a break, with $P(1,76)$ 
$=11.56$. The only other significant term from the analywis was the interaction between conctituent break type and serial ponitions, where $F(2,76)=3.87$. From Fis. 1 , it appean that the effects of different constituent break pocitions decreased when clicks occurred late in the sentence.

The test for migration towrards the major clause breal was concerned only with the condition in which the click was either at a minor breal or not at a break. In order to maximize the probalility of obtaining an effect, the data were scored in the same way as that reported by Bever, Leckner, and Kirk (1969). Not only were the accurate responsen exchuded from the malyim, bat also excladed were recponse which were beyond the major break ("orershoots"). Reeponses which were not in the direction of the break but were disploced by an amount greater than the distence between the break and the objective click location were alwo excluded. For each $S$, the number of rexponses going toward or into the mijor clave break wros expressed as a proportion of the number of remaining errots. The overall mean proportion of migration erron was 576. On the actumption that a majority of the erron specified should go towats of into the mojor clanue breal, the appropriatie test was to compare thi value with 5 , asiug the error mean square (MASI) calculated from an andyais of vriance. This test showed that .576 wo sipnifienutly higher than .5 , with $F(1,38)=6.63$, indicating that a swall, but significant, mifration effect had oceurred.

That repposes mignted towards the breal from clich poritions so far anry from it exemed surpriating. However, a post hoce andysin shomed thet the only mean from the fon relent conditions wich differed Einifienth from .5 wes thet for cifele which were not at a brealt and were late in the semtence, with $F(3,33)$ $=9.73$. An expinendion for this reml" is implied by Indefored and Brond bent (19e0) and more resently bs Bertidnon and Thesre (1970) who reported that arels hend dining sentences were jodpad acenring prior to theit extan lockions Seventy-ifve percent of all bection emors in the preat experinest pleeed the click prior to its oljective locotion. This preposition tendenes creatie a polbiem for intergretion the wiontion efiect, since all early eicels which were not at a breal cocommed before the mior breal, while all bite clicks which were not at a breal followed the mojor break. Throx, preposition and wipation were confounded in the very condition which post hoc analyais showed to be the source of the migration effect.

\section{EXPLRIMLNT 2}

This experiment aimed to balance the preposition tendency with the migration tendency by placing clicks both before and after the major clause break, thereby demonstrating an effect of syntactic structure on click location accuracy apart from a migration effect. It was hypothesized that migration towards major clause breaks would not oceur and that location aceuracy would remoin higher for clictss at a major clawe break than for clicks not at a break. Agpin, large separations of the chick and the break were employed.

Clicks were beated either in the irst half (early) or in the second half (late) of 16 sentences which were 15 words long. Chels were also placed either at the major elanese break or not at a break. For clicks not at a break, half the clicks were before the clause break and half were after it. The average separation of such clicks from the major clause break was 7.1 syllables.

\section{Subject:}

Twenty graduate atudents and meanbers of staff in the Department of Prychology at the Univerity of Melboume acted as $\mathrm{S}$.

\section{Rewilk}

Of all the exroneons click location judgments, $84 \%$ were reconded prior to the objective click location. Migration wa caleulated in the sume way as above: the obtoined proportion of .571 erros mignting toward the major clause break was not significantly different from .5 , with $F(1,19)=2.94$. Anoming that migntion stomld dimppen when the clidt and the bre-l are widely ceponted, the fince to find a mipention effoct wo anticipated. However, it is porithe for an effect to be obveimed by a ling amomet of variance. Setiting $a=0=.05$, an experiment hawine two obsentions on each of $20 \mathrm{Br} \mathrm{cm}$ deteet a difference from 5 of .151, with 1838 $=0685$. This vilse is and withion the mevitude of the difference (.27) reported by Bever, Lneloner, and Kink (1969), who found .77 erros in frwor of mistion wing the same reoring procedire with ctidis very close to the buent. Thes, the prepent experiment

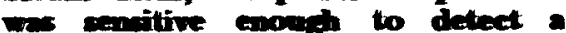
mipration effect equel in mogitude to thet previoudy reportied, aithong a sunlle migntion tenderey mes leve gone undetected. The point we to demondinte not that migntion efifects do not occur at all, but that differences in the accuracy of click location may occur independently of significant migration.

The mean proportions of accurate judgments over So were as follows: .438 for early clicks at a break, .388 for late chicks at a break, .125 for early clicks not at a break, and .100 for late clicks not at a break. Analysis of variance of the scores showed that the only significant effect was constituent break type, with $F(1,19)=22.61$. Thus, it has been shown that location aceuracy was significantly better for clicks at major clause breaks than for clicks not at such breaks, in the absence of migration.

\section{EXPIRIMENT 3}

This experiment was designed to assess the effect of the requirement that $S 6$ reproduce the sentence in the click location talk. The obvious reason for instructing $S$ to write out the sentence is to force him to process and retain the sentence while trying to judige the location of the click. However, it may be the ease that writing out the sentence alters, or even creates, errors in click location. Therefore, a situation was devieed where Ss were forced to procen the sentence but did not alwayn hove to wite it ont. Two respone conditions were ned: either Si bad to reproduce the sentence, or they were anpplied with a correct vernion after the sentence hod been presented. These two conditions were interspenied randomity for a given S. The second variable of interest was the separation of the chict from the nujor clanse break. It hos been ansumed that the tendency for migration tomard the breal would be greater the neaver the elick was to the breal. Two

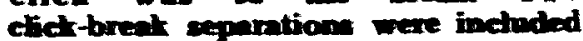
in the experinent to give a pretinings inditiofion of eng effects on cilluer migration or necures.

Thenty aentences were propended in

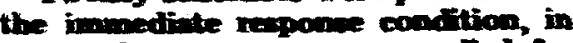
which the ecatence wre soppried for the $S^{\prime}$ and 20 sentences were presented in the delared reopore condition, in which $S$ hod to repodince the centense. All 10 sentenses were 14 wond lons and vere comerneted wo that a mijor anfiace structume breals cecmend after the sementh wond and sepuratied the entence into two

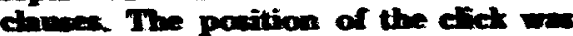
the some for ench respone condition. In 4 sentencos, the etiol occurred at the mojor alme break; in 16, the cited wo not a a breal. In the hittes sentences, the chich cecurned in syllwe prior to the bret, two syllabe prior to the breal, sx 


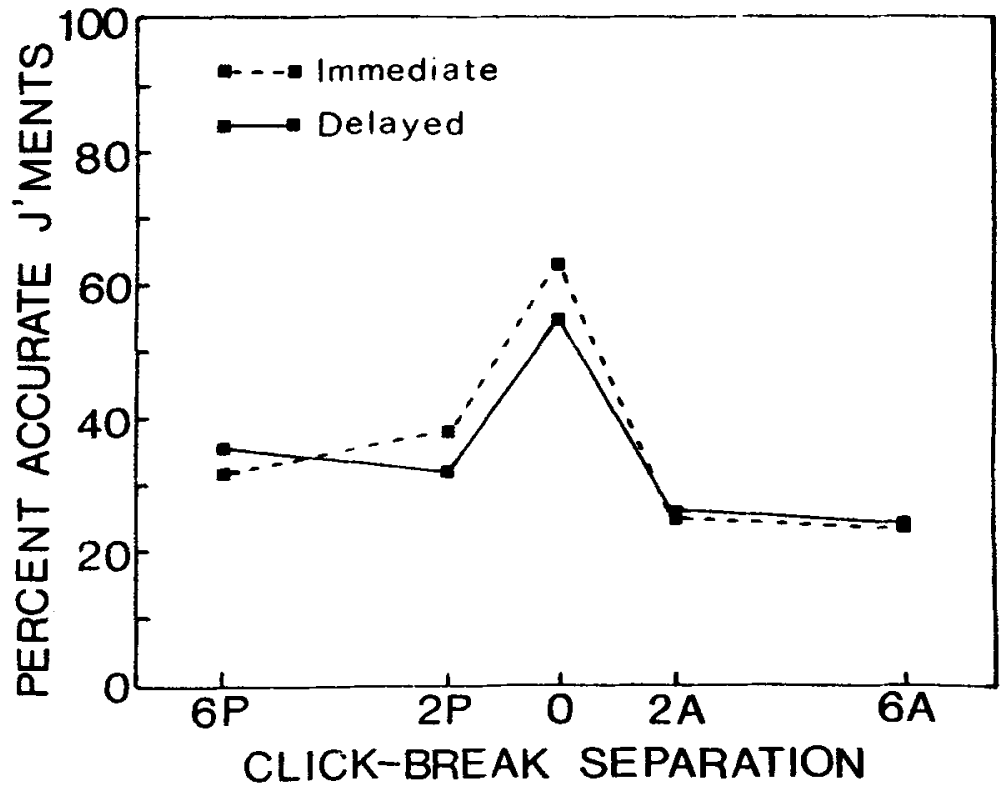

Fig. 2. Percentage of accurate click location judgments in Experiment 3 as a function of the separation (in syllables) between the click and the major clause break and of immediate compared with delayed responding.

syllables after the break, or two syllables after the break. The five locations are shown by slashes in the following example: Just after John ordered/ his new/ car/, he/ saw a/ better bargain somewhere else. Response booklets were prepared in such a way that $S$ knew as soon as he turned the page at the end of each recorded sentence whether to write out the sentence before marking the location of the click.

\section{Subjects}

Thirty-eight undergraduate students from the University of Melbourne, who were paid for their participation, acted as Ss. The data for one $S$ were rejected after it was found that he had recorded judgments only in one response condition.

\section{Results}

The mean percent of accurate judgments for the various conditions is shown in Fig. 2, where little difference between the two response conditions can be observed. Analysis of variance of the scores showed that the response condition factor was not significant, with $F(1,36)=0.59$. The SS for click-break separation and response condition were divided into six orthogonal contrasts, leaving a residual with two degrees of freedom. The first two contrasts compared location accuracy at the break with the four not-at-break conditions. The contrasts were significant in both response conditions: for delayed responding, $F(1,144)=33.75$, and for immediate responding, $F(1,144)=26.49$. Significantly more errors were made for clicks located after the break than before the break, with $F(1,144)=6.49$ for delayed responding, and with responding. The final two contrasts compared performance for clicks two syllables from the break with that for clicks six syllables from the break. For neither delayed responding, where $F(1,144)=0.16$, nor immediate responding, where $F(1,144)=1.39$, was there a significant effect. The results support those found in Experiments 1 and 2 and show that increase the probability of forgetting the available click location information.

The mean proportions of errors $F(1,144)=8.63$ for immediate reproduction of the sentence does not migrating towards or into the major clause break are shown in Table 1 . Analysis of variance of the data showed that there was no significant effect due to delayed or immediate responding, with $F(1,36)=0.14$. The SS for click-break separation plus Click-Break Separation by Response Condition were again divided into six components, four of which were orthogonal contrasts and two of which were noncontrast hypotheses. There was no significant difference between migration for clicks six or two syllables from the break: for delayed responding, $F(1,108)=0.08$, and for immediate responding, $F(1,108)=$ 0.04 . However, migration was significantly greater for clicks after the break than before the break: for delayed responding, $F(1,108)=55.56$; and for immediate responding, $F(1,108)=33.21$. The two noncontrast hypotheses tested whether the proportion of migration errors both before and after the break were different from .5. After the break, the proportion was significantly greater than .5 , with $F(1,108)=$ 120.73 , while before the break it was significantly less than .5 , with $F(1,108)=4.71$.

The results of this experiment demonstrate that Ss' having to reproduce the sentence affects neither the accuracy of judgments of click location nor the tendency for location judgments to migrate towards the syntactic break. Accuracy was highest for clicks at the major clause boundary in both response conditions, independent of the migration effects. While before-break clicks migrated less than after-break clicks, they were still located much less accurately than clicks at the break. Finally, the failure to show that click-break separation affected migration argues against the assumption that migration should increase as clicks occur closer to the break.

\section{EXPERIMENT 4}

The aim of this experiment was to evaluate more precisely the effect of the separation of the click from the major clause break. The reason that no effect of click-break separation on either migration or accuracy was found in Experiment 3 might be that the relation is a nonlinear one. Since
Table 1

Mean Proportion of Click Location Errors Migrating Tow ard or into the Major Clause Break in Experiment 3

\begin{tabular}{lcccc}
\hline & \multicolumn{5}{c}{ Click-Break } & Separation \\
\cline { 2 - 5 } $\begin{array}{l}\text { Response } \\
\text { Condition }\end{array}$ & 6 Prior & 2 Prior & 2 After & 6 After \\
\hline Delayed & .385 & .464 & .806 & .854 \\
Immediate & .495 & .419 & .798 & .743 \\
\hline
\end{tabular}

Table 2

Mean Proportion of Click Location Errors Migrating Towand or into the $M$ ajor Clause Break in Experiment 4

\begin{tabular}{lcccc}
\hline & \multicolumn{5}{c}{ Click-Break } & Separation & \\
\cline { 2 - 5 } $\begin{array}{l}\text { Position } \\
\text { of Click }\end{array}$ & 9 & 6 & 4 & 2 \\
\hline Prior to Break & .233 & .304 & .400 & .408 \\
After Break & .917 & .908 & .867 & .808 \\
\hline
\end{tabular}


more than two conditions are required to detect such a trend, a larger number of click-break separations were used in this experiment.

\section{Design}

Twenty-seven 14-word sentences were constructed with one-syllable words in the 5 th to 10 th positions (i.e., the middle 6 words) and with two-syllable words in the 4th and 11th positions. The first and last 3 words varied in syllable length. There were three sentences in each of nine conditions of click-break separation: the click was either at the major clause break (which was always after the seventh word) or at nine, six, four, or two syllables prior to or after the break. Thus, the clicks were located after the 3rd, 4th, 5th, 6th, 7th, 8th, 9th, 10th, or 11th words. The following sentence illustrates the possible click positions: Because it was/ snowing/ at/ the/ lodge/, we/ left/ our/ luggage/ in the hotel.

\section{Subjects}

Forty students from an undergraduate psychology course at the University of Melbourne volunteered to be Ss.

\section{Results}

Figure 3 shows the mean percent accurate responses as a function of click-break separation. In the analysis of variance, the eight degrees of freedom were taken up by the following tests. First, the location of clicks occurring at the major clause break was judged significantly more accurately than the location of clicks at all other positions, with $F(1,312)=$ 70.44. Secondly, the comparison of the conditions with the click prior to the break and after the break was also significant, with $F(1,312)=21.02$. The third to eighth contrasts tested three components of trend for clicks prior to and after the break. There was a significant linear trend for responses both prior to the break, with $F(1,312)$ $=15.57$, and after the break, with $F(1,312)=11.07$. The quadratic and cubic components did not approach significance. Thus, the accuracy of the judgments was dependent on click-break separation; fewer accurate judgments were made when the click was close to the break.

The mean proportions of migration errors are shown in Table 2. Three components of trend were tested for responses to clicks prior to and after the break. The only significant component was a linear trend for clicks prior to the break, with $F(1,273)=6.57$. The linear trend for clicks after the break was not significant, with $\mathrm{F}(1,273)=1.29$; $\mathbf{F}<1$ for all other tests. The proportion of migration errors prior to

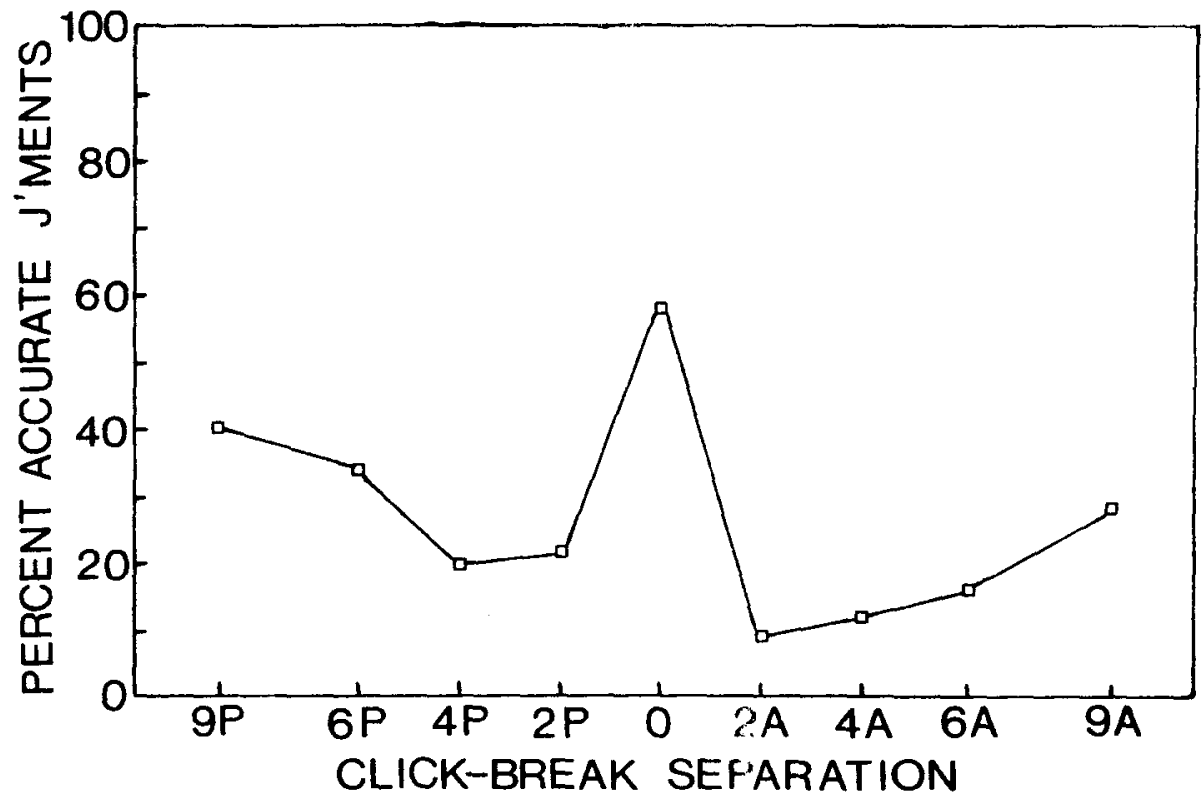

Fig. 3. Percentage of accurate click location judgments in Experiment 4 as a function of the separation (in syllables) between the click and the major clause break.

the break was significantly lower than .5 , with $F(1,273)=36.47$; the proportion of migration errors after the break was significantly greater than .5 , with $F(1,273)=191.47$.

The results show that as click-break separation decreased, Ss' judgments to before-break clicks became less accurate, apparently due to an increasing tendency to locate the clicks towards the major clause break. The fact that the proportion of migrating before-break clicks was a minority, however, demonstrates that the tendency to prepose the clicks is even stronger as a determinant of the location judgments. For after-break clicks, the picture is not so clear. While Ss did become less accurate in locating the clicks as the distance between the click and the break decreased, there was no corresponding increase in the proportion of errors migrating towards the break. The only plausible explanation of these results for after-break clicks seems to be that the preposing tendency is stronger than the migration tendency. That is, any increase in the tendency for the break to attract errors as click-break separation decreases might be counteracted by a decrease in the tendency to locate the clicks prior to their objective positions, as clicks become closer to the beginning of the sentence. Thus, the preposing tendency might have prevented the detection of an increasing trend in the migration effect for after-break clicks.

\section{DISCUSSION}

The results of Experiment 1 have shown that accuracy of click location varies as a function of the position of the click in the constituent structure of sentences. Clicks were found to be located most accurately at the boundaries between major clauses, with intermediate accuracy between major phrases within clauses, and least accurately within simple phrases. Experiments 2-4 were designed to provide support for the claim that these results can be explained by during sentence recognition. In Experiment 2, it was shown that such location results obtain under conditions where there is no detectable click migration towards the major clause break. Thus, differences in accuracy of click location are not necessarily dependent on migration effects. The results of Experiment 3 demonstrated that the pattern of click location judgments obtained is the same when $S$ is required to reproduce the sentence as when he is not required to do so. This is an important confirmation of the view that accuracy of click location reflects perceptual processing load.

The fact that the separation of the click from the major clause break influences accuracy of click location, as found in Experiment 4, suggests that location accuracy and migration are not always independent. Therefore, in tests of hypotheses about finer aspects of structure than those features examined in the present experiments, the distance of the click from the break may have to be controlled. However, the trends in accuracy of click location as a function of click-break separation variations in processing difficulty 
were small compared with the differences obtained between different positions in the constituent structure. Thus, this result does not seriously challenge the basic finding of Experiment 1.

It should be stressed that the failure to find a significant tendency for judgments of click location to migrate towards major clause breaks in Experiment 2, and the partial confirmations of migration in Experiments 3 and 4, is not necessarily inconsistent with prior research. The aim of the present experiments was to set up conditions where migration would be minimal. Previous investigations, in which a migration effect has been clearly demonstrated, have all used click positions near the relevant constituent break, where the migration effect is strongest. Because it was not the purpose of Experiments 3 and 4 to detect a migration effect, the tendency to prepose judgments was left confounded with the tendency for judgments to migrate. While the evidence from these experiments suggests that the preposing tendency is a stronger determinant of the direction of the responses than migration, this conclusion may not apply in other experimental situations.

Given that accuracy of click location is a valid index of sentence processing load, the question to be asked is: At what stage in processing of the sentence do the lcation errors arise? The main result of Experiment 1 is in strong agreement with that of Holmes and Forster (1970), who found that reaction times (RTs) were faster to clicks at a major clause break than to clicks at a minor break or not at a break. This result indicates that the high location accuracy at the major clause break is at least partly due to the reduction in attention necessary for processing the sentence at the time such clicks occur.

The results of Holmes and Forster (1970) and those of the present experiments are not parallel in every respect. First, the difference between RTs to clicks at a minor break and clicks not at a break was weak and inconsistent in Holmes and Forster (1970), whereas location accuracy for these click positions was substantially different in the present experiments. This implies that, although attention to sentence processing may not differ reliably within major clauses according to minor constituent structure, subsequent processing into these minor constituents must occur. Secondly, RTs in Holmes and Forster (1970) were markedly faster to clicks late in the sentence than to early clicks. In Experiments 1 and 2, location accuracy was no higher for late than for early clicks. (In fact, in Experiments 3 and 4, early clicks were more accurately located than late clicks. This probably reflects the confounding of the preposition and the migration tendencies.) Hence, the absence of an analogous serial position effect for location accuracy implies that judgments of click location are influenced by processing input subsequent to the click.

The differences between the results of Holmes and Forster (1970) and those of the present experiments indicate that additional processing must affect judgments of click location, but they do not lead to the conclusion that "the psychological mechanism for reacting to clicks is distinct from the mechanism for locating clicks [Abrams \& Bever, 1969, p. 286]." Abrams and Bever placed clicks in the syllable prior to the major clause break, at the break, or in the first syllable after the break. They reported that "Although before-break clicks receive slower RT than after-break clicks, in-break clicks do not receive the fastest RT [1969, p. 283]" and inferred that "attention to non-speech activity is reduced at the ends of clauses rather than enhanced [ 1969 , p. 287]." However, in their two experiments, the mean of the RTs to clicks before and after the break was almost the same as that for RTs to clicks in the break. That after-break RTs were faster than before-break RTs might as plausibly be explained by a decreasing serial position effect as by an effect of syntactic structure. Moreover, placing the clicks for the three conditions so close to one another would not only make it difficult to detect reliable differences in RTs, but also would make the conditions different from those in Holmes and Forster (1970), in which large click-break separations were used. Thus, Abrams and Bever's conclusion about the relation of RTs and location accuracy is based on quite different evidence from that considered in our experiments.

In conjunction with the results of Holmes and Forster (1970), the present findings suggest the following view of the processes operating during the click location task. A marked reduction in attention to sentence processing at major clause boundaries, which produces comparatively fast RTs, allows clicks to be more accurately located there than at other parts of the sentence. While it is not clear whether attention varies within major clauses, judgments of click location are subject to less interference from subsequent sentence processing at minor breaks within these clauses than within phrases. This implies that the words of sentences may not be coded into phrases immediately as they are heard but may be recoded into major phrases for storage in short-term memory (at least where verbatim recall is required). The general preposing tendency found in the experiments may result from S's delaying his coding of the sentential input slightly by making use of his echoic storage system (Neisser, 1967). The click, on the other hand, has to be dealt with rapidly before it is forgotten and so tends to be judged in relation to the objectively earlier input which the $S$ is now processing.

The processes described attempt to explain the determinants of accuracy of click location, but they do not preclude the possibility of a migration effect occurring. In Bever, Lackner, and Kirk (1969) and Bever (1970), it has been argued that the tendency for migration arises from a very early stage in processing, as a result of an initial perceptual segmentation strategy. ${ }^{6}$ However, it seems to us that migration errors are more likely to arise from later stages in processing. If interference from the requirements of sentence processing has made the $S$ unclear as to the location of the click, then the more processing he is required to do, the more likely is the $S$ to integrate his location response with concurrent processing. Hence, S may erroneously locate the click towards the boundary of a unit he is recoding in short-term memory. This might lead one to expect a tendency for clicks to be located towards minor constituent boundaries as well as towards major clause boundaries. However, this is a difficult hypothesis to test, owing to the problems in constructing criteria for assessing the strength of the effects at minor breaks. The available evidence does not support the prediction that clicks migrate towards minor breaks (Bever, Lackner, \& Kirk, 1969).

A final issue needing further investigation is whether it is essential for the major constituent break to be a boundary between S-dominated constituents (clauses) in order for there to be the greatest possible reduction in processing load. Correspondingly, it would be interesting to know whether minor breaks in the sentence structure produce decreased processing load more effectively if they are clause rather than phrase boundaries.

\section{REFERENCES}

ABRAMS, K., \& BEVER, T. G. Syntactic structure modifies attention during speech perception and recognition. Quarterly Journal of Experimental Psychology, 1969, 21, 280-290.

BERTELSON, P., \& TISSEYRE, F Perceiving the sequence of speech and nonspeech stimuli. Quarterly Journal of 
Experimental Psychology, 1970, 22, $653-662$.

BEVER, $T$. $G$. The cognitive basis for linguistic structures. In J. R. Hayes (Ed.), Cognition and the development of language. New York: Wiley, 1970.

BEVER, T, KIRK, R \& LACKNER, J. An autonomic reflection of syntactic structure. Neuropsychologia, 1969, 7 . 23-28.

BEV ER, T, G., LACKNER, J. R., \& KIRK, $R$. The underlying structures of sentences are the primary units of immediate speech processing. Perception \& Psychophysics, 1969, 5, 225-234.

BEVER, T. G., LACKNER, J. R., \& STOLZ, W. Transitional probability is not a general mechanism for the segmentation of speech. Journal of Experimental Psychology, 1969, 79, 387-394.

FODOR, J. A., \& BEVER, T. G. The psychological reality of linguistic segments. Journal of Verbal Learning \& Verbal Behavior, 1965, 4, 414-420.

GARRETT, M., BEVER, T. G., \& FODOR, J. A. The active use of grammar in speech perception. Perception \& Psychophysics, 1966, 1, 30-32.

HAYS, W. I. Statistics for psychologists. New York: Holt, Rinehart \& Winston. 1963.
HOLMES, V. M. \& FORSTER, K. I Detection of extraneous signals during sentence recognition. Perception \& Psychophysics, 1970, 7, 297-301.

LADEFOGED, P., \& BROADBENT, D. E. Perception of sequence in auditory events. Quarteriy Journal of Experimental Psychology, 1960, 1.2. $162-170$.

NFISSER, U. Cognitive psychology. New York: Appleton-Century-Crofts, 1967

\section{NOTES}

1. A clause is defined for present purposes as a surface constituent, except an adjective, dominated by an $S$ in the underlying structure.

2. A complete list of the sentences used in each experiment, together with the mean click location accuracy and mean migration scores for each sentence, can be obtained from ASIS National Auxiliary Publications Service (c/o CCM Information Sciences, Inc., 22 West 34th Street, New York, New York 10001).

3. It could be argued that two of the sentences do not fit the classification exactly. In one sentence (Sentence 14 in Holmes \& Forster, 1970), where the click is said to be at a minor break, the click is between two NPs, but may interrupt more constituents than some of those in the not-at-break condition. In another (Sentence 19 in Holmes \& Forster, 1970), where the click is said to be not at a break, the click may not interrupt as many constituents as others in this condition.

4. All subsequent decisions were based on a Type I error rate of $\alpha=.05$.

5. A general preposing tendency was not discussed by Garrett et al (1966). Bever. Lackner, and Kirk (1969), and Bever, Lackner, and Stolz (1969). Bever, Kirk and Lackner (1969) found that shocks presented during sentences were judged to occur a fraction of a syllable prior to their objective location. However, Fodor and Bever (1965) found almost no overall preposition effect: $52 \%$ of the error responses were placed prior to the objective location and $48 \%$ were placed after the objective location.

6. Abrams and Bever have also claimed that "the systematic nature of errors in click location are due to perceptual processing which follows the initial segmentation of the speech stimulus [1969, p. 288]." This view is more compatible with the explanations expressed in the present paper.

Accepted for publication January 27, 1972. 